\title{
Análise eletromiográfica do músculo deltoide em diferentes posições de contração isométrica voluntária máxima
}

http://dx.doi.org/10.11606/1807-5509202000020205

\author{
Cláudia Silveira LIMA* \\ Bruno Tomasi KUCKARTZ* \\ Cleiton Silva CORREA* \\ Cristine MARSICO* \\ Rafael Cristane MICHEL* \\ Ronei Silveira PINTO* \\ *Laboratório de \\ Pesquisa do Exercício, \\ Escola de Educação \\ Física, Universidade \\ Federal do Rio Grande \\ do Sul, Porto Alegre, \\ RS, Brasil.
}

\section{Resumo}

Diferentes posições articulares são utilizadas para realizar a contração isométrica voluntária máxima (CIVM) que servirá de referência para normalização do sinal eletromiográfico (EMG), porém em muitas destas posições não se verifica a máxima ativação do músculo deltoide. 0 objetivo deste estudo foi comparar a atividade elétrica das três partes deste músculo em diferentes posições articulares durante CIVMs e determinar em quais dessas são obtidas as maiores ativações musculares, para utilização na normalização do sinal EMG. A amostra foi constituida de 12 indivíduos do sexo masculino com idades entre 20 e 30 anos, treinados em força. Foi mensurada a ativação a partir da utilização da eletromiografia de superfície em seis posições articulares para a parte clavicular do músculo deltoide e em mais seis posições articulares para as partes acromial e espinal. Foi calculada, posteriormente, o valor RMS do sinal EMG de cada porção do músculo deltóide (clavicular, acromial e espinal). Para comparar essas ativações em relação ao fator posição, que inclui as seis posições de CIVM avaliadas, aplicou-se ANOVA de medidas repetidas, com teste post-hoc LSD, adotando um nível de significância de 5\%. Para a parte clavicular do músculo deltoide, a posição de maior valor RMS foi em pé com flexão de ombro a $90^{\circ}$ e realizando uma flexão do ombro; para as partes espinal e acromial foi em pé com abdução de ombro a $90^{\circ}$ e executando uma extensão horizontal. Assim, estas posições articulares parecem ser as mais propícias para mensuração da máxima ativação das três partes do músculo deltoide durante as CIVMs, sendo adequada a utilização do sinal EMG obtido nestas condições para o posterior processo de normalização.

PalaVRAS-CHAve: Eletromiografia; Ombro; Força Muscular; Músculo Esquelético.

\section{Introdução}

A eletromiografia tem sido uma das técnicas mais utilizadas na avaliação da participação muscular em diferentes condições, sendo bem reconhecida a necessidade da realização da normalização para a adequada comparação de diferentes músculos e indivíduos. O processo de normalização deve ser realizado a partir do valor máximo do sinal eletromiográfico (EMG) de cada músculo, determinando à ativação relativa do mesmo músculo em diferentes exercícios, dinâmicos ou isométricos ${ }^{1}$. Sem a adequada normalização dos dados EMG, não é possível efetuar comparaçôes confiáveis entre diferentes músculos, indivíduos ou exercícios ${ }^{2}$.
Uma das formas mais utilizadas para o processo de normalização dos dados EMG envolve a utilização de exercícios isométricos, com os quais é possível obter-se a ativação máxima ou quase máxima dos músculos de interesse ${ }^{3-5}$. Além disso, eles são facilmente executados e possibilitam a coleta do sinal EMG em condiçôes de maior estabilidade ${ }^{6}$. No entanto, os processos de normalização nem sempre são realizados na condição na qual se verifica maior nível de ativação muscular ${ }^{7}$. Preconiza-se que os estudos utilizem posições articulares e funçôes musculares onde o músculo a ser testado seja um dos principais agonistas do movimento, tendo assim 
maior efetividade para alcançar o nível máximo de ativação muscular. A falta de padronização na determinação das posições de realização das CIVMs pode alterar de forma significativa os valores relativos de ativação muscular após o processo de normalização (i.e. valores mais baixos do sinal EMG obtido durante as CIVMs podem superestimar os valores relativos) $)^{8,9}$.

$\mathrm{Na}$ literatura científica percebesse uma diversidade de posiçôes para normalização dos dados EMG de um mesmo músculo ou grupo muscular, tanto em membros inferiores ${ }^{10,11}$ quanto em membros superiores $^{12,13}$. Em relação ao músculo deltoide, um estudo ${ }^{12}$ adotou apenas uma única posição para a normalização do sinal EMG das três partes do musculo deltoide (ombro em $90^{\circ}$ nos planos frontal e escapular), realizando nesta posição a contração máxima de abdução. Neste estudo, não foram consideradas as demais funçóes deste musculo. Diferentemente, em outro estudo ${ }^{13} \mathrm{em}$ que também foi avaliada a atividade eletromiografia deste musculo, a posição do ombro abduzido em

\section{Método}

A amostra foi constituída por 12 sujeitos do sexo masculino com idade entre 20 e 30 anos, com no mínimo seis meses de experiência em treino de treino de força e inscritos, voluntariamente, no processo de seleção da amostra.

O cálculo amostral foi baseado no estudo de Welsch, Bird e MAYHeW ${ }^{14}$ devido à semelhança com as avaliaçóes realizadas no presente estudo. $\mathrm{O}$ cálculo foi realizado para amostras emparelhadas através do programa PEPI versão 4.0, em que foi adotado um nível de significância de 0,05 , um poder de $80 \%$, e um coeficiente de correlação de 0,7 para todas as variáveis.

\section{Procedimentos de Coleta de Dados}

Cada indivíduo realizou duas sessóes de coletas de dados na sala de musculação da Escola de Educação Física, Fisioterapia e Dança da Universidade Federal do Rio Grande do Sul (ESEFID/UFRGS), tendo dois dias de intervalo entre as sessôes.

$\mathrm{Na}$ primeira sessão foram esclarecidas todas as etapas do estudo e realizada a assinatura do Termo de Consentimento Livre e Esclarecido, aprovado pelo Comitê de Ética em Pesquisa da Universidade Federal do Rio Grande do Sul, pelo número 2008012. $90^{\circ}$ foi adotada para a avaliação da flexão horizontal e extensão horizontal do ombro, sendo a posição de $90^{\circ}$ de flexão do ombro adotada para a avaliação da função de flexão desta articulação; ou seja, mais de uma posição foi adotada no processo de normalização do sinal EMG. Assim, observa-se diferentes estratégias de posição utilizadas em alguns estudos, bem como também a ausência desta importante informação em outros ${ }^{14-18}$.

Diante da dificuldade de encontrar na literatura científica a adoção de um padrão relativo à posição do ombro adotada no processo de normalização do sinal EMG quando o deltoide é o músculo de interesse, bem como a não consideração das diferentes funçôes das partes deste músculo (clavicular, acromial e espinal) neste processo, o presente estudo tem como objetivo comparar a atividade elétrica das três partes do músculo deltoide em diferentes posições articulares durante CIVMs e descrever as melhores posições e funçôes a serem adotadas no processo de normalização do sinal EMG do músculo deltoide.

Ainda na primeira sessão, os sujeitos foram submetidos às CIVMs em seis diferentes posiçóes para a aquisiçáo do sinal EMG da parte clavicular do deltoide. Além da posição a ser avaliada também foi selecionada, entre todas as funçóes do músculo deltoide (flexão, flexão horizontal, abdução e extensão horizontal), as principais funçôes cinéticas exercidas pela parte clavicular do músculo: flexão e flexão horinzontal. As posiçóes selecionadas para realizar a CIVM do músculo na função de flexão foram: (1) Em pé, com flexão de ombro de $45^{\circ}$ (F45) e (2) Em pé, com flexão de ombro em $90^{\circ}$ (F90). As posiçóes selecionadas para testar o músculo na função de flexão horizontal foram: (1) Em pé, com ombro abduzido em $90^{\circ}(\mathrm{FH})$; (2) Em pé com ombro abduzido em $90^{\circ}$ e $45^{\circ}$ de flexão horizontal (FHD); (3) Decúbito dorsal com ombro abduzido em 90 (FHS) e (4) Decúbito dorsal com abduçáo de ombro em $90^{\circ}$ e cotovelo flexionado em $90^{\circ}$ (FHF) (FIGURA 1).

$\mathrm{Na}$ segunda sessão foram realizadas as CIVMs para as partes acromial e espinal do músculo deltoide e, novamente, as posiçóes foram selecionadas de acordo com as açóes principais correspondentes a essas partes do músculo: abdução e extensão horizontal. As posições selecionadas para contração isométrica do músculo na 
função de abdução foram: (1) Em pé, com abdução de ombro de $135^{\circ}$ (A13) e (2) Em pé, com abdução de ombro em $90^{\circ}$ (A90). As posições selecionadas para testar o músculo na função de extensão horizontal foram: (1) Em pé, com ombro abduzido em $90^{\circ}$ (EH9); (2) Em pé com ombro abduzido em $90^{\circ}$ e $45^{\circ}$ de flexão horizontal (EHD); (3) Decúbito ventral com ombro abduzido em $90^{\circ}$ (EHV) e (4) Decúbito ventral com ombro abduzido em $90^{\circ}$ e com cotovelo flexionado em $90^{\circ}$ (EHF) (FIGURA 2).

A ordem de execução dos exercícios, realizados nas duas sessôes, foi randomizada para todos os indivíduos e o intervalo de tempo entre cada CIVM foi de no mínimo cinco minutos, de modo a minimizar os efeitos da fadiga no sinal $\mathrm{EMG}^{19}$.

Para a aquisição dos dados das CIVMs, cada indivíduo realizou três contraçóes isométricas voluntárias máximas em cada uma das 12 posiçóes articulares citadas anteriormente. Cada CIVM foi realizada durante um período de cinco segundos ${ }^{20}$. Durante o período de realização das CIVMs, além do sinal EMG, também obteve-se o sinal correspondente à curva de força com a utilizaçáo de uma célula de carga acoplada ao equipamento.

\section{Aquisição do Sinal EMG}

Para a aquisição dos dados eletromiográficos utilizouse um eletromiógrafo Miotool 400, da marca MIOTEC - Equipamentos Biomédicos (Porto Alegre, Brasil). O equipamento é composto por um sistema de 4 canais, $2000 \mathrm{~Hz}$ por canal. Foram utilizados eletrodos de superfície, com $15 \mathrm{~mm}$ de raio, pré-amplificados com configuração bipolar da marca Tyco Healthcare, Mini Medi-Trace 100, Kendall Medtrace.

Antes da colocação dos eletrodos realizou-se a tricotomia e a abrasão da pele com algodão eálcool, com o intuito de diminuir a impedância da pele. $\mathrm{O}$ nível de resistência dos eletrodos foi medido antes de cada sessáo com um multímetro digital (Brasfort - Brasil), sendo aceitos valores de até $3000 \mathrm{Ohms}$. Os eletrodos foram posicionados no ventre muscular das partes clavicular, acromial e espinal do músculo deltoide21 e a distância intereletrodos foi de $20 \mathrm{~mm}$ entre os centros de cada eletrodo. $\mathrm{O}$ eletrodo terra foi posicionado na clavícula.

\section{Aquisição da Curva de Força}

A curva de força foi registrada com a utilização de uma célula de carga (marca Miotec - Equipamentos
Biomédicos, Porto Alegre, Brasil, resolução de 1 kgf) acoplada ao sistema de peso do equipamento de musculação. A célula de carga foi conectada a um conversor analógico digital (A/D) Miotool (Miotec-Equipamentos Biomédicos, Porto Alegre, Brasil) em um dos canais do equipamento de eletromiografia, o que garantiu a coleta sincronizada do sinal EMG e da curva de força.

A curva de força foi utilizada para identificar o momento de estabilização da produçáo de força, ou seja, o platô desta curva. Neste período é que foi recortada a janela de um segundo do sinal EMG para cálculo do valor RMS.

\section{Análise dos Dados}

Os sinais captados pelo eletromiógrafo, referentes à ativação muscular e à curva de força, foram gravados em um microcomputador no software Miograph, e posteriormente analisados no software SAD32.

Para a análise dos dados, foi realizada a filtragem digital do sinal EMG utilizando-se filtros do tipo Passabanda Butterworth, de $5^{\mathrm{a}}$ ordem, com freqüência de corte entre 20 e $500 \mathrm{~Hz}$. Após os procedimentos de filtragem descritos acima, as curvas do sinal EMG e de força correspondente às contraçóes voluntárias máximas (tempo de 5 segundos) foram recortadas. Dentro deste período de 5 segundos, foi identificado o platô da curva de força e realizado o recorte de uma janela de um segundo durante esse platô. Os valores RMS foram calculados neste intervalo, sendo o maior valor dentre as três CIVMs de cada indivíduo considerado para posterior análise estatística dos dados em cada exercício.

\section{Análise Estatística}

A fim de se verificar a normalidade e a esfericidade dos dados, aplicaram-se, respectivamente, os testes de Shapiro-Wilk e de Mauchly. Ao passo que foi identificado que os dados apresentaram distribuição paramétrica, os resultados foram expressos em média e desvio-padrão. Para comparar as ativaçóes de cada porção do músculo deltóide (clavicular, acromial e espinal) em relação ao fator posição, que inclui as seis posiçóes de CIVM avaliadas, aplicou-se ANOVA de medidas repetidas, com teste post-hoc LSD. O nível de significância adotado foi de 0,05 e o programa estatístico utilizado foi o SPSS, versão 15.0. 


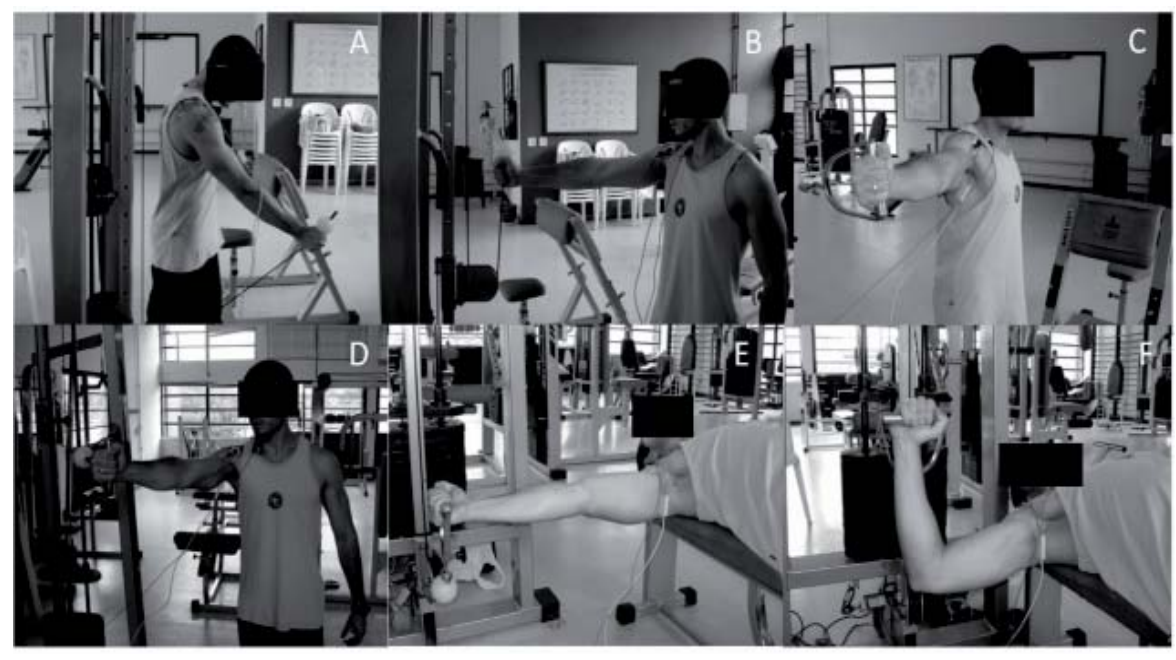

FIGURA 1 - Funções e posições analisadas para a parte clavicular do músculo deltoide: A) Flexão 45ํㅜ (F45), B) Flexão $90^{\circ}$ (F90), C) Flexão Horizontal com 90o deabdução (FH), D) Flexão Horizontal com 90ํ de abdução e 45으 deflexão horizontal (FHD), E) Flexão horizontal em decúbito dorsal com 90o abdução (FHS) eF) Flexão horizontal em decúbito dorsal com 90ํ deabdução e90ํㅓㅁ deflexão decotovelo (FHF).

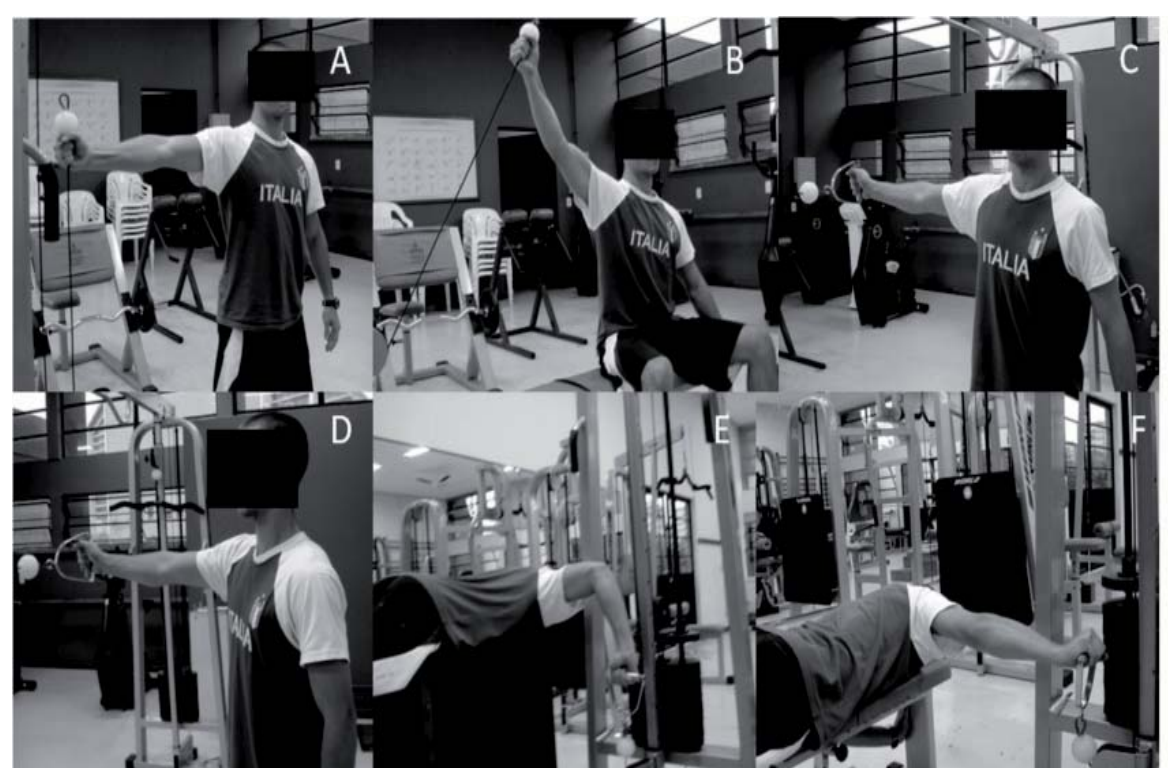

FIGURA 2 - Funções e posições analisadas para as partes acromial e espinal do músculo deltoide: A) Abdução de ombro a 90ำ (A90), B) Abdução de ombro a 135o (A13), C) Extensão horizontal com abdução de

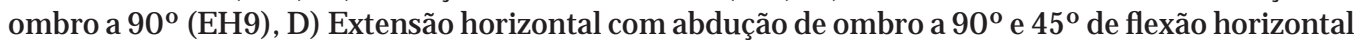
(EHD), E) Extensão horizontal em decúbito ventral com abdução de ombro a 90o eflexão de cotovelo a 90ํㅡ (EHF) e F) Extensão horizontal em decúbito ventral com abdução de ombro a 90ํ EHV). 


\section{Resultados}

Inicialmente os dados serão apresentados de forma descritiva por percentagem, para cada uma das partes do deltoide. O percentual máximo de ativação $(100 \%)$ foi considerado para a posição com maior valor RMS e os níveis de ativação das demais posiçóes foram estabelecidos como percentual relativo à posição de maior atividade eletromiográfica (TABELA 1).

TABELA 1- Percentual de ativação da parte clavicular, acromial e espinal do músculo deltoide em cada posição, considerada a posição de maior ativação como máxima (100\%).

F45 - flexão do ombro a $45^{\circ}$;

F90 - flexão do ombro a $90^{\circ}$;

FH - flexão horizontal com 0 ombro abduzido a $90^{\circ}$; FHD - flexão horizontal com o ombro abduzido a $90^{\circ}$ e flexão horizontal de $45^{\circ}$;

FHF - flexão horizontal em decúbito dorsal com o ombro abduzido a $90^{\circ} \mathrm{e}$ com o cotovelo flexionado a $90^{\circ}$;

FHS - flexão horizontal em decúbito dorsal com 0 ombro abduzido a $90^{\circ}$. A13 - abdução de ombro a $135^{\circ}$,

A90 - abdução de ombro a $90^{\circ}$;

EH9-extensão horizontal com o ombro abduzido a $90^{\circ}$;

EHD - extensão horizontal com o ombro abduzido a $90^{\circ} \mathrm{com} 45^{\circ}$ de flexão horizontal;

EHF-extensão horizontal em decúbito ventral com o ombro abduzido a $90^{\circ} \mathrm{e}$ com o cotovelo flexionado a $90^{\circ} \mathrm{e}$

EHV-extensão horizontal em decúbito ventral com 0 ombro abduzido a $90^{\circ}$.

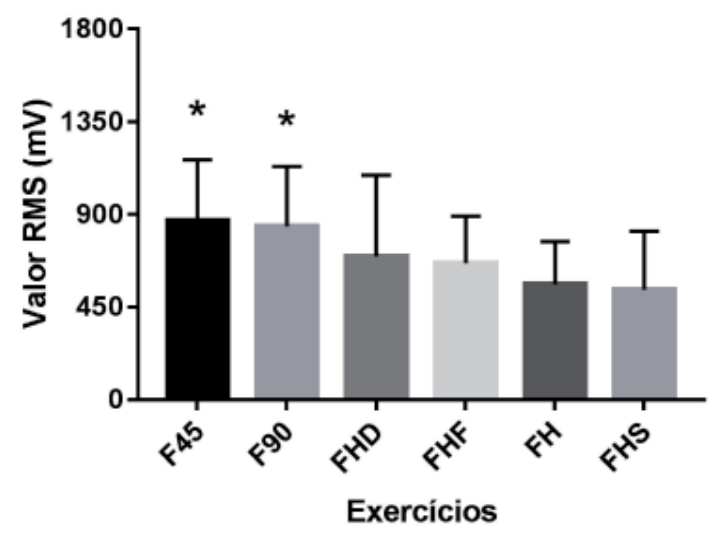

* = diferença significativa em relação ao FHD, FHF FH e FHS $(p<0,05)$.

FIGURA 3 - Valor RMS do músculo deltoide (parte clavicular) nas posições flexão do ombro a 45 (F45), flexão do ombro a $90^{\circ}$ (F90), flexão horizontal com o ombro abduzido a $90^{\circ}$ e flexão horizontal de $45^{\circ}$ (FHD), flexão horizontal em decúbito dorsal com o ombro abduzido a $90^{\circ}$ ecom o cotovelo flexionado a $90^{\circ}$ (FHF), flexão horizontal com o ombro abduzido a $90^{\circ}(\mathrm{FH})$ e flexão horizontal em decúbito dorsal com o ombro abduzido a 90ํ (FHS). 
* $=$ diferença significativa das demais condições $(\mathrm{p}<0,05)$.
* = diferença significativa em relação ao A90 e A13 $(p<0,05)$.

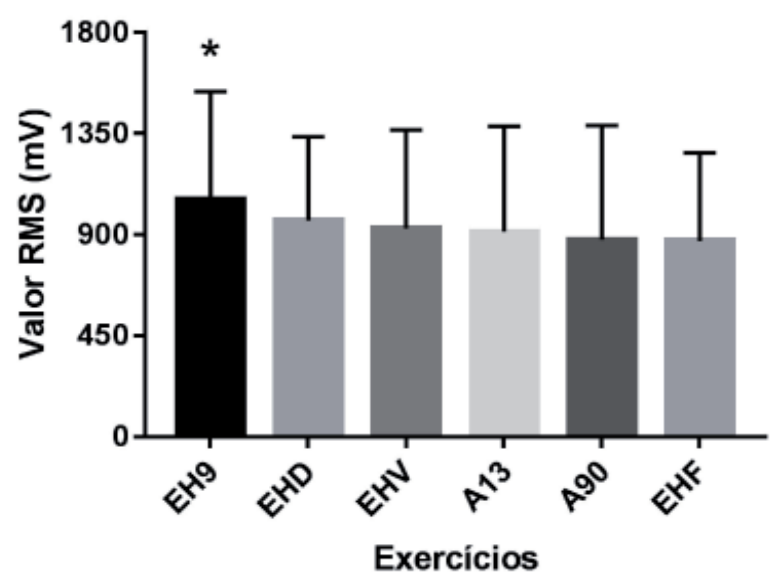

FIGURA 4 - Valor RMS do músculo deltoide (parte acromial) nas posições extensão horizontal com o ombro abduzido a $90^{\circ}$ (EH9), extensão horizontal com o ombro abduzido a $90^{\circ} \mathrm{com} 45^{\circ}$ deflexão horizontal (EHD), extensão horizontal em decúbito ventral com o ombro abduzido a $90^{\circ}$ (EHV), abdução de ombro a $135^{\circ}$ (A13), abdução de ombro a $90^{\circ}$ (A90) e extensão horizontal em decúbito ventral com o ombro abduzido a $90^{\circ} \mathrm{com}$ o cotovelo flexionado a $90^{\circ}$ (EHF).

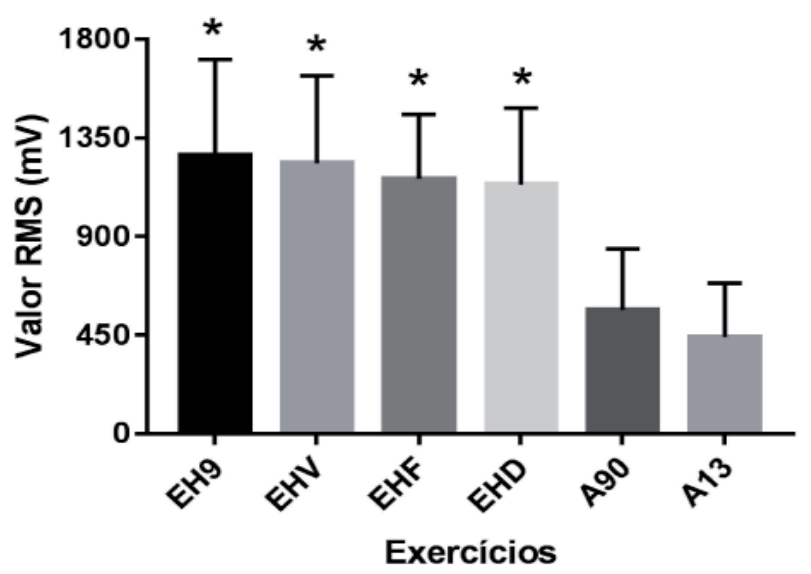

FIGURA 5 - Valor RMS do músculo deltoide (parte espinal) nas posições extensão horizontal com o ombro abduzido a $90^{\circ}$ (EH9), extensão horizontal em decúbito ventral com o ombro abduzido a $90^{\circ}$ (EHV), extensão horizontal em decúbito ventral com o ombro abduzido a $90^{\circ} \mathrm{com}$ o cotovelo flexionado a $90^{\circ}$ (EHF), extensão horizontal com o ombro abduzido a $90^{\circ} \mathrm{com}$ flexão horizontal a $45^{\circ}$ (EHD), abdução do ombro a $90^{\circ}$ (A90) e abdução do ombro a $135^{\circ}$ (A13).

\section{Discussão}

Ao analisar os resultados do presente estudo com $\mathrm{o}$ intuito de averiguar as melhores posiçóes e funções a serem adotadas no processo de normalizaçáo dos sinais eletromiográficos das diferentes partes do músculo deltoide observou-se que para parte clavicular do músculo deltoide as posiçóes com o ombro em flexão e na função de flexão produziram maior atividade eletromiográfica. Para parte acromial a posição de abdução do ombro testando a função de extensão horizontal foi a de maior ativaçáo. E, para parte espinal qualquer das posiçôes de abdução testando a função de extensão horizontal foram as de maior atividade elétrica muscular. Estes resultados demonstram que não só a posição articular mas também a função 
avaliada são determinantes para a mensuração da máxima ativação muscular.

A parte clavicular do músculo deltoide demonstrou maior nível de ativação nas posições com o ombro flexionado realizando a ação de flexão quando comparada à posição de abdução do ombro em $90^{\circ}$ realizando a ação de flexão horizontal. Estes resultados corroboram com outro estudo ${ }^{22}$ que, embora seja um estudo voltado para análise de fadiga da parte clavicular e espinal do deltoide, relatou que a parte clavicular do deltoide com o ombro na posição de $90^{\circ}$ de flexão e em contração isométrica para manter a posição apresentou maior ativação do que a parte espinal do deltoide na mesma situação. Os autores atribuíram a maior ativação EMG da parte clavicular do deltoide, por ser ela considerada agonista do movimento, e assim é responsável por uma porcentagem maior de trabalho e, portanto, um maior número de unidades motoras precisa se ativado. Além disso, a parte clavicular do músculo deltoide apresentou maior atividade em uma das posiçóes (flexão) em que o músculo é considerado motor primário do movimento ${ }^{23}$. Embora esta parte do deltoide também seja considerada motora primária da função de flexão horizontal, a menor ativação deste músculo durante os testes de flexão horizontal de ombro pode ser explicada pelo sinergismo do músculo deltoide parte clavicular com os músculos peitoral maior e coracobraquial, dividindo a ação de flexão horizontal do ombro com estes músculos, enquanto que na flexáo de ombro ele executa esta função apenas com o auxílio da parte clavicular do peitoral maior ${ }^{24}$.

Para a parte acromial do músculo deltoide, o maior nível de ativação observado na ação de extensão horizontal do ombro não era esperado, uma vez que esta parte do músculo, apesar de ser definida na literatura ${ }^{23}$ também como motora primária da extensão horizontal, na maioria dos estudos a posição utilizada para obtenção do sinal EMG da CIVM é em abdução de $90^{\circ}$, só que testando a ação de abdução, sabidamente a função primária mais conhecida desta parte do músculo deltoide ${ }^{23}$. Os resultados do presente estudo possibilitam especular que a função de extensão horizontal do ombro seria mais importante do que a função de abdução. No entanto, este maior valor ao testar a ação de extensão horizontal, pode ser decorrente da associação das ações de abdução, que nesta posição é solicitada para sustentação do segmento na posição horizontal e a função de extensão horizontal efetivamente testada no movimento. A necessidade de atuação para mais de uma função primária ao mesmo tempo parece demandar maior atividade muscular da parte acromial do músculo do que quando ele realiza as funçôes separadamente. A idéia de que a demanda muscular aumenta nas situações em que o músculo realiza mais de uma função pelas quais ele é responsável já foi evidenciada ${ }^{25}$, porém em relação ao músculo gastrocnêmio. Corroborando com o exposto anteriormente, outro estudo ${ }^{13}$ apresenta a maior ativação do músculo deltoide parte acromial na ação isométrica de abdução do ombro em 90 na posição em pé, quando comparada à abdução do ombro a $90^{\circ}$ em decúbito ventral (ambos testando a extensão horizontal). Os autores sugerem que a elevada atividade elétrica desse músculo na posição de abdução de ombro a $90^{\circ}$ em pé, pode ser explicada pela necessidade de maior recrutamento de unidades motoras para que o músculo possa manter a posição, tendo em vista que a parte acromial do deltoide deve sustentar o peso do braço nessa posição, o que não ocorre na posição de decúbito ventral, na qual o músculo deltoide parte espinal realiza a tarefa de sustentação do peso do segmento.

Para o músculo deltoide parte espinal, todas as posiçóes em que o ombro se encontra em ângulo de $90^{\circ}$ e a ação testada é a de extensão horizontal apresentaram valores RMS significativamente maiores que as posiçóes onde foi testada a ação de abdução do ombro. Estes dados demonstram que, independente da posição, a maior atividade do músculo deltoide parte espinal ocorre na função de extensão horizontal quando comparada à função de abdução, o que encontra respaldo na proposição de $\mathrm{RASCH}^{24}$, segundo a qual a parte espinal do deltoide tem como função primária a extensão horizontal do ombro e secundária a abdução do ombro. Os resultados do presente estudo apontam que, independente dos ângulos de abdução do ombro e flexão do cotovelo, as posiçóes em que é avaliada a função de extensão horizontal do ombro, a ativação da parte espinal do deltoide é máxima.

Considerando os resultados do presente estudo, as seguintes posiçôes articulares para avaliação das partes clavicular, acromial e espinal do deltoide durante as CIVMs são propostas: parte clavicular, posição articular de flexão de ombro à $90^{\circ}$, tendo em vista que esta posição ajuda na execução do teste por facilitar o posicionamento dos indivíduos e a aferição do ângulo de execução do exercício; partes acromial e espinal, posição em pé com ombros abduzidos a $90^{\circ}$ e executando a ação de extensão horizontal, ao utilizar uma única posição para testar as partes 
acromial e espinal do deltoide, além de facilitar o procedimento de coleta, podem evitar problemas como a fadiga muscular e deslocamentos dos eletrodo ocasionados pelas mudanças de posição. Outro aspecto a ser salientado é que os dados obtidos, tanto para a parte acromial quanto para a espinal, podem ser normalizados exatamente nas mesmas condiçóes e situaçóes.

Como limitação do estudo, embora tenha sido dado um intervalo importante $(5 \mathrm{~min}$ ) entre as CIVMs e as posições testadas tenham sido randomizadas, a presença da fadiga pode ter afetado a magnitude do sinal EMG e, consequentemente, os resultados e conclusóes deste estudo.

Os resultados apresentados nesse estudo apontaram as posiçôes com o ombro em flexão que testaram a funçáo de flexáo do ombro como sendo as que possibilitaram os maiores níveis de atividade EMG da parte clavicular do músculo deltoide. A posição de abdução de $90^{\circ}$ do ombro realizando a açáo de extensão horizontal, por sua vez, gerou maior ativação da parte acromial do músculo deltoide. Todas as posiçôes com o ombro em ângulo de $90^{\circ}$ realizando a ação de extensão horizontal produzem maior atividade mioelétrica da parte espinal do músculo deltoide. Os resultados permitem ainda concluir que para otimizar as coletas de dados com vistas à normalização de dados EMG originados no músculo deltoide a posição de abdução de $90^{\circ}$ do ombro realizando a ação de extensão horizontal pode ser utilizada para a coleta do sinal EMG da CIVM tanto para parte acromial quanto para espinal deste músculo. Esses resultados permitem uma escolha mais precisa das posiçóes em que há maior ativação dos músculos avaliados.

\title{
Conflito de interesse
}

Os autores não apresentam conflito de interesse.

\begin{abstract}
Electromyographic analysis of the deltoid muscle in differents positions of maximum voluntary isometric contractions

Different joint positions are used to perform maximum voluntary isometric contractions (MVIC) in order to normalize electromyographical (EMG) signals, even though most of them are not the ones with the greatest deltoid muscle electric activation. The aim of this study is compare the electric activity of the three parts of the deltoid muscle in different joint positions during MVIC and examine the best positions to use in normalization of the EMG signals. Twelve strenght-trained men with ages between 20 and 30 years volunteered to the study. Surface EMG signals were recorded in six joint positions for the clavicular part and six positions for the acromial and spinal parts of the deltoid muscle. The RMS value of the EMG signal of each portion of the deltoid muscle (clavicular, acromial and spinal) was subsequently calculated. To compare these activations in relation to the position factor, which includes the six MVIC positions evaluated, a repeated measures ANOVA was applied with a post-hoc LSD test and a significance level of 5\% was set. For the clavicular part of the deltoid muscle, the position of greatest RMS value was with the subject standing with shoulder flexion at $90^{\circ}$ and performing a flexion movement. For the acromial and spinal parts of the deltoid muscle was the standing position with shoulder abduction at $90^{\circ}$ and performing a horizontal extension. Therefore, these joint positions seen to be more propitious to measure the maximum activation of the three parts of the deltoid muscle during the MVIC, being more adequate the utilization of the EMG signal obtained in these conditions for a posterior process of normalization.
\end{abstract}

KEYwORDS: Electromyography; Shoulder; Muscle Strength; Skeletal Muscle. 


\section{Referências}

1. Hodder J, Keir P. Obtaining maximum muscle excitation for normalizing shoulder electromyography in dynamic contractions. Journal of Electromyography and Kinesiology 2013;23(5):1166-73.

2. Burnnet A, et al. Examination of EMG normalization methods for the study of the posterior and posterolateral neck muscles in healthy controls. J Electromyogr Kinesiol 2007;17(5):635-41.

3. Jakobi JM, Chilibeck PD. Bilateral and unilateral contractions: Possible differences in maximal voluntary force. Canadian Journal of Applied Physiology 2001;26(1):12-33.

4. Häkkinen K, Kraemer WJ, Newton RU. Muscle activation and force production during bilateral and unilateral concentric and isometric contractions of the knee extensors in men and women at different ages. Electromyogr Clin Neurophysiol 1997;37(3):131-42.

5. De Luca CJ. The use of surface electromyography in biomechanics. J Appl Biomec 1997;13:135-63

6. Burden A. How should we normalize electromyograms obtained from healthy participants? What we have learned from over 25 years of research. J Electromyogr Kinesiol. 2010;20(6):1023-35.

7. Intenlangelo L., Bordachar D, Barbosa AW. Effects of scapular taping in Young adults with shoulder pain and scapular dyskinesis. J Bodyw Mov Ther. 2016;20(3):525-32

8. Gurney A, et al. Shoulder Electromyography Measurements during Activities of Daily Living and Routine Rehabilitation Exercises. J Orthop Sports Phys Ther. 2016;46(5):375-83.

9. Nakamura Y, Tsuruike M, Ellenbecker TS. Electromyographic Activity of Scapular Muscle Control in Free-motion Exercise. J Athl Train. 2016;51(3):195-204.

10. Gondin J, Guette M, Martin BA. Neural and muscular changes to detraining after electrostimulation. European Journal of Applied Physiology 2006;97(2):165-73.

11. Kurunganti U, Murphy T. Bilateral deficit expressions and myoelectric signal activity during submaximal and maximal isometric knee extensions in young, athletic males. European Journal of Applied Physiology 2008;102(6):721-26.

12. Rodrigues D, Pessan MA, Kawano MM, Stabile GR, Cardoso JR. Electromyographic analysis of deltoid muscle fatigue during abduction on scapular and frontal planes. Electromyogr. Clin. Neurophysiol 2008;48(6-7):293-300.

13. Oliveira AS, Freitas CMS, Monaretti FH, Ferreira F, Noguti R, Bérzin F. Avaliação eletromiográfica de músculos da cintura escapular e braço durante exercícios com carga axial e rotacional. Revista Brasileira de Medicina do Esporte 2006;12(1):11-5.

14. Welsch EA, Bird M, Mayhew JL. Electromyographic activity of the pectoralis major and anterior deltoid muscles during three upper-body lifts. Journal of Strenght and Conditioning Research 2005;19(2):449-52.

15. Gioftsos G, et al. EMG activity of the serratus anterior and trapezius muscles during the different phases of the push-up plus exercise on different support surfaces and different hand positions. J Phys Ther Sci. 2016;28(7):2114-8.

16. Kinali G, Kara S, Yildirim MS. Electromyographic analysis of an ergonomic risk factor: overhead work. J Phys Ther Sci. 2016 Jun;28(6):1924-7.

17. Kim E, Oh J, Yoo W. Effect of Vibration Frequency on Serratus Anterior Muscle Activity during Performance of the Push-up Plus with a Redcord Sling. J Phys Ther Sci. 2014;26:1275-1276.

18. Jeong SY, Chung SH, Shim JH. Comparison of Upper Trapezius, Anterior Deltoid, and Serratus Anterior Muscle Activity during Push-up Plus Exercise on Slings and a Stable Surface. J Phys Ther Sci. 2014;26:937-939.

19. Kuorinka I. Restitution of EMG spectrum after muscular fatigue. Eur. J. Appl. Physiol 1988;57:311-315.

20. Leis AA, Trapani VC. Atlas of electromyography. Oxford, New York: Oxford, University Press, 2000.

21. Gnitecki JE, Kler GPS, Moussavi Z. EMG signs of fatigue in anterior and posterior deltoid muscles: questioning the role of RMS during fatigue. Department of Electrical \& Computer Engineering University of Manitoba, Winnipeg, Manitoba, R3T 2N2, Canada. 2000.

22. Schenkman M, Cartaya V. Kinesiology of the Shoulder Complex. The Journal of Orthopaedic and Sports Physical Therapy 1987;8(9):438-50.

23. Smith LK, Wess EL, Lehmukuhl LD. Cinesiologia clínica de Brunnstrom. São Paulo: Manole, 1997.

24. Rasch PJ, Burke RK. Cinesiologia e Anatomia Aplicada. Philadelphia: Guanabara Koogan, 1977.

25. Signorile J, et al. Selective recruitment of the triceps surae muscles with changes in knee angle. The Journal of Strength \& Conditioning Research 2002; 16(3):433-439. 
Lima CS, et al.

ENDEREÇO Cláudia Silveira Lima

Rua Felizardo, 750

90690-200 - Porto Alegre - RS - BRASIL E-mail: claudia.lima@ufrgs.br
Recebido para publicação: 25/ 05/ 2016

1a. Revisão: 23/ 03/2016

2a. Revisão: $22 / 03 / 2017$

Aceito: 29/06/2017 